International Journal of Engineering \& Technology, $7(2.33)(2018) 607-610$
International Journal of Engineering \& Technology
WPC
Website: www.sciencepubco.com/index.php/IJET
Research paper

\title{
Modern shopping cart with automatic billing system using load sensor
}

\author{
D. Kavitha * \\ Faculty, Computer Science and Engineering, Sri Sai Ram Engineering College, Chennai, Tamil Nadu, India \\ *Corresponding author E-mail: Kavithad.cse@sairam.edu.in
}

\begin{abstract}
A survey of people's shopping habits has been completed recently and they've come up with the top 10 list of the things that people hate when supermarket shopping. These figures were taken from a survey of 2500 people on their supermarket shopping habits. There is no surprise on the number one issue - It was long queues at the check-outs with 62 per cent of the people surveyed stating this to be their big issue when shopping in a super market. So we have enhanced an idea to avoid long queues by providing SMART TROLLEY. Here we have provided a barcode reader and digital display in order to display the total amount of the shopping. It has two additional functionalities to make this Trolley more efficient. It has a LOAD CELL to detect the mal practice. In addition, we have provided IN SWITCH and OUT SWITCH for customer ease of use.
\end{abstract}

Keywords: Barcode Reader; LCD Display; RS-232; Micro-Controller; Trolley

\section{Introduction}

Purchasing and shopping at big malls is becoming daily activity in metro cities. After completion of purchases, to avoid long queues in supermarket shopping which is the biggest issue that people hate in supermarket shopping. To save the time of the customers in supermarket we have introduced SMART TROLLEY to overcome the delay which usually takes place in supermarket billing system especially in special occasions or in festival seasons.

\section{Literature survey}

The article discusses the usage of mobile phones to monitor shopping time at physical stores. A phone-based shopping tracker transforms the problem of monitoring shopping time into a classification problem. It uses motif groups to identify movement trajectories based on spatial and temporal features embedded in each motif. [1] Traditionally 'Shopping' is a phenomenon where a customer and the merchant meet face to face in order to exchange goods and services for a payment, in a live environment; that experience cannot be virtualized by online-shopping which cannot give the real feeling of shopping and can create more disadvantages rather than the advantages it introduces. According to observations regarding realtime shopping where you can get the real experience, the solution developed as a mobile phone application on the Android platform would help the customer go to the shelf that he wants by seeing the indication on an indoor map designed in it. [2]

Activity monitoring, a crucial task in many applications, is often conducted expensively using video cameras. Effectively monitoring a large field by analyzing images from multiple cameras remains a challenging issue. Other approaches generally require the tracking objects to attach special devices, which are infeasible in many scenarios. The RFID technology provides an economically attractive solution due to the low cost of RF tags and readers. Another novelty of this design is that the tracking objects do not need to be equipped with any RF transmitters or receivers.[3]

Indoor human activity recognition remains a hot topic and receives tremendous research efforts during the last few decades. However, previous solutions either rely on special hardware, or demand the cooperation of subjects. Therefore, the scalability issue remains a great challenge. To this end, we present an online activity recognition system, which explores Wi-Fi ambient signals for received signal strength indicator (RSSI) fingerprint of different activities. It can be integrated into any existing WLAN networks without additional hardware support. In addition, it does not need the subjects to be cooperative during the recognition process. [4]

\section{Existing system}

At present, the humans are needed to provide a billing for each grocery. This results in queue in order to provide a bill for each and every customer. In order to avoid the waiting time of customer in the queue, here we provide alternate solution by providing new automatic billing system So that would in turn reduce long queue.

\section{Proposed system}

In our proposed system we are introducing SMART TROLLEY based automated billing and payment system .A unique ID is given for each trolley . Here, we provide Barcode for each grocery. INSWITCH is default so the customers can scan the items and place inside the trolley. And in order to take out the items from the trolley, they need to press the OUT SWITCH. Based on this the billed items will be cancelled. Finally these details will be automatically sent to the billing unit through WSN. Here the data will be automatically uploaded to the billing section. And the workers or a staff will able 
to see the each consumer purchase and the billing amount. The consumer will easily make its payment whenever they reach the billing section. Depends on the consumer purchase, the amount will be notified on LCD. In order to detect the mal practice we are using a LOADCELL. Weight of the things inside the trolley will be calculated separately and checked with weight of items scanned by barcode reader. Server will compare both the weights. Incase these two weights didn't match then malpractice had occurred somewhere then that specific customer had to check separately. If both the weights matched then customer can just collect their shopping items from the counter and they can move without wasting their time for collecting bill and checking the items.

\section{Advantages}

- The main aim of our project is to avoid long queues in supermarket shopping which is the biggest issue that people hate in supermarket shopping.

- To save the time of the customers in supermarket we have introduced SMART TROLLEY to overcome the delay which usually takes place in supermarket billing system especially in special occasions or in festival seasons.

\section{System design}

\subsection{Block diagram}

Trolley Section:

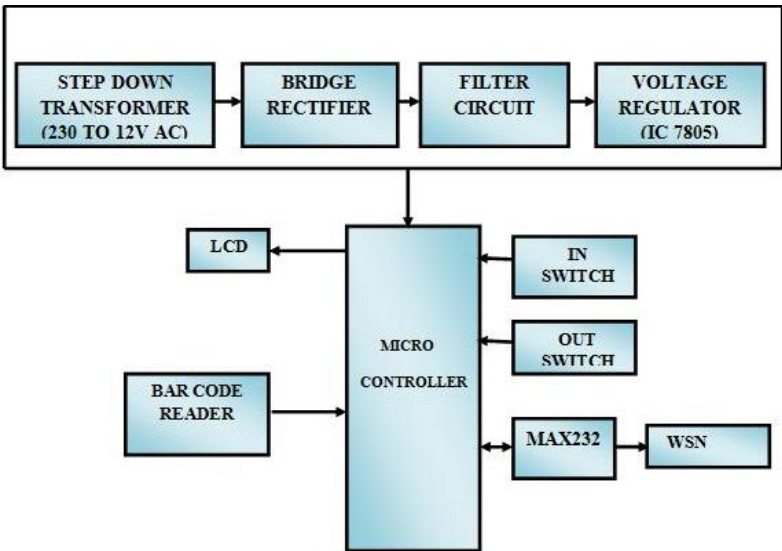

Fig. 1: Hardware Implementation of Modern Shopping Cart.

Billing Section:

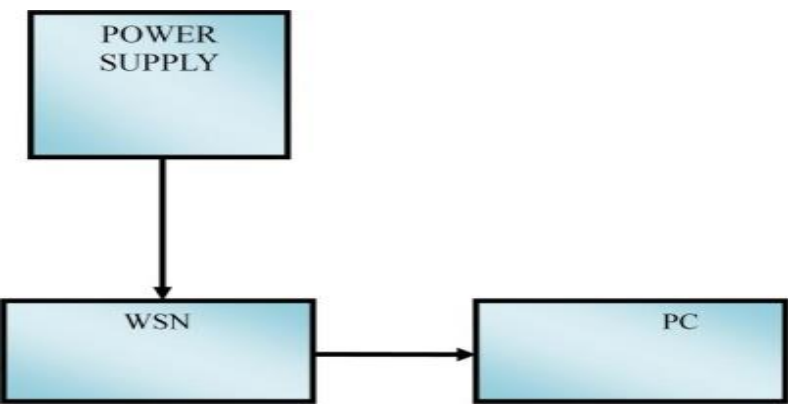

Fig. 2: Billing Section Cart to Shop PC.

\subsection{Microcontroller (PIC16F877A)}

In single chip all functions are performed. Microcontroller is a general purpose chip it is used to create a device or multifunction computer; it requires multiple chips to handle different tasks. A microcontroller is used to be more self-contained, functions as a tiny, independent and dedicated computer. PIC16f877a finds its applications in a huge number of devices. It is used in remote sensors, security and safety devices, home automation and in many industrial instruments. An EEPROM is also featured in it which makes it possible to store some of the information permanently like transmitter codes and receiver frequencies and some other related data. The cost of this controller is low and its handling is also easy. Its flexible and can be used in areas where microcontrollers have never been used before as in coprocessor applications and timer functions etc.

\subsection{Max 232 (serial interface)}

RS232 is meant for Serial transmission and it involves sending of data one bit at a time, on a single communication line. In contrast, parallel communication require as many lines as there are bits in a word being transmitted (for an 8-bit word, a minimum of 8 lines are needed). For long distance communication purpose serial transmission is used whereas parallel transmission is used for short distance communication and also providing high transmission rate. Information being transferred between data processing equipment and peripherals is in the form of digital data which is transmitted in either a serial or parallel mode. Parallel communications are used mainly for connections between test instruments or computers and printers, while serial is often used between computers and other peripherals.

\subsection{Barcode reader}

It consists of a light source, a lens and a light sensor translating optical impulses into electrical ones This scanner is meant to scan the products which are usually at a distance about 3 to 8 inches from the symbol and this is done automatically when the trigger is pulled off.

\subsection{Liquid crystal display}

A liquid crystal display is an electronically modulated device that is commonly used for displaying purpose and the most widely used LCSs that are available in the market are 1 Line, 2 Line or 4 Line LCD s that are having only one microcontroller and they are capable of supporting more than 80 characters when they make use of 2 HD44780controllers. Most LCDs that support only one controller has 14 Pins whereas LCDs that support 2 controller has 16 Pins in which both the type of microcontrollers were provided with two extra pins that is for back-light LED connections.

\subsection{In switch (add button) / out switch (delete button)}

Switch is an electrical component that can break an electrical circuit, interrupting the current or diverting it from one conductor to another. The mechanism of a switch may be operated directly by a human operator to control a circuit (for example, a light switch or a keyboard button), may be operated by a moving object such as a door-operated switch, or may be operated by some sensing element for pressure, temperature or flow. A relay is a switch that is operated by electricity. Switches are made to handle a wide range of voltages and currents; very large switches may be used to isolate high-voltage circuits in electrical substations.

\subsection{Wireless sensor network}

(WSNs) can be defined as a self-configured and infrastructure less wireless networks to monitor physical or environmental conditions, such as temperature, sound, vibration, pressure, motion or pollutants and to cooperatively pass their data through the network to a main location or sink where the data can be observed and analysed. A base station acts like an interface between users and the network. One can retrieve required information from the network by injecting queries and gathering results from the skin.

Typically a wireless sensor network contains hundreds of thousands of sensor nodes. A wireless sensor node is equipped with sensing and computing devices, radio transceivers and power components. The individual nodes in a wireless sensor network (WSN) are inherently resource constrained: they have limited processing speed, 
storage capacity, and communication bandwidth. After the sensor nodes are deployed, they are responsible for self-organizing an appropriate network infrastructure often with multi-hop communication with them. Then the onboard sensors start collecting information of interest. Wireless sensor devices also respond to queries sent from a "control site" to perform specific instructions or provide sensing samples. The working mode of the sensor nodes may be either continuous or event driven. Global Positioning System (GPS) and local positioning algorithms can be used to obtain location and positioning information. Wireless sensor devices can be equipped with actuators to "act" upon certain conditions.

\subsection{Comparing weights to detect malpractice}

\section{LOAD SENSOR}

A load cell is a transducer that is used to create an electrical signal whose magnitude is directly proportional to the force being measured. The various types of load cells include hydraulic load cells, pneumatic load cells and strain gauge load cells. This load cell will compare the weight of products generated in the cart to the weight generated in the server side. If the weight matches then the user can directly pay the bill amount. If the weight mismatch then mal practise had occurred.

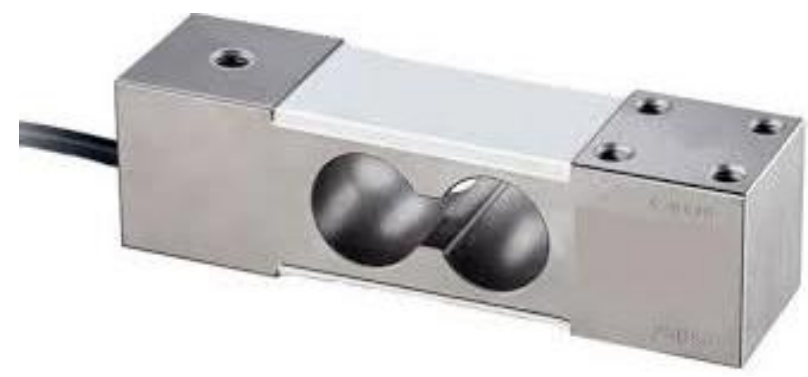

Fig: 3: Load Sensor to Detect Malpractice.

\section{Implementation}

- Scanning product

- Displaying amount

- Interfacing with server using RS232

- Bill generation

- Load Balance checker

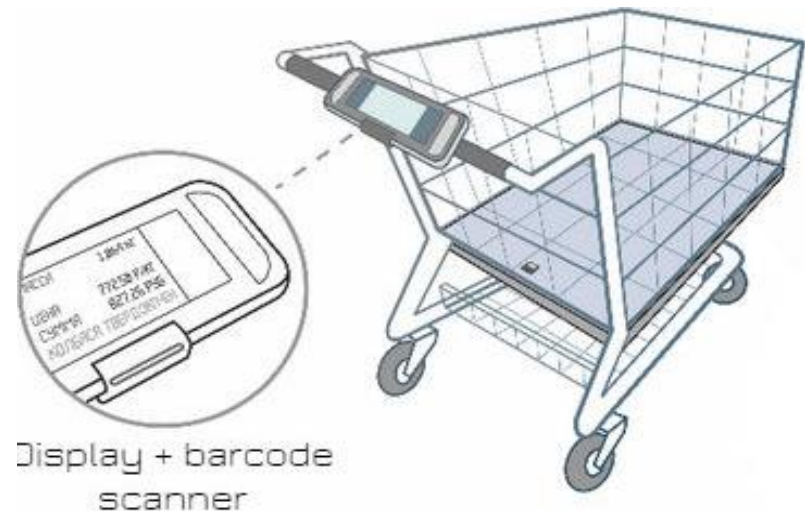

Fig. 4: Modern Shopping Cart with Barcode Scanner and LCD Display.

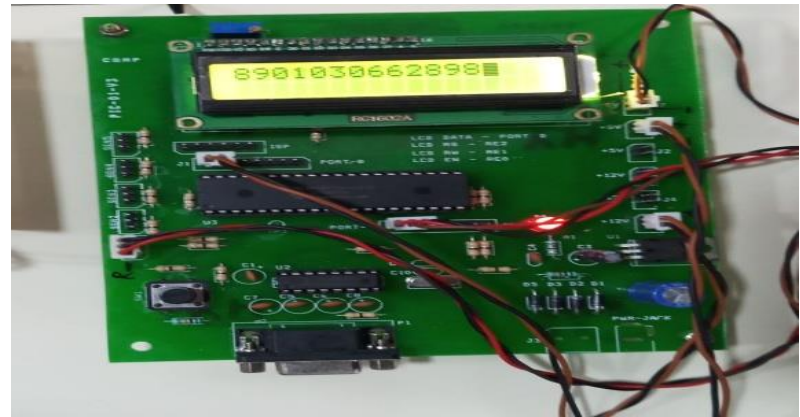

Fig. 5: Displaying the Barcode of the Product after Barcode Scan.

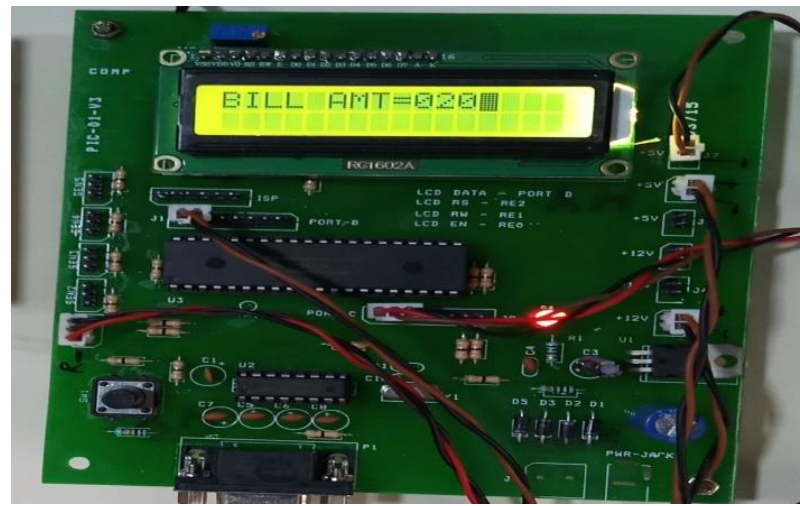

Fig. 6: Displaying the Amount after Product Added to Cart.

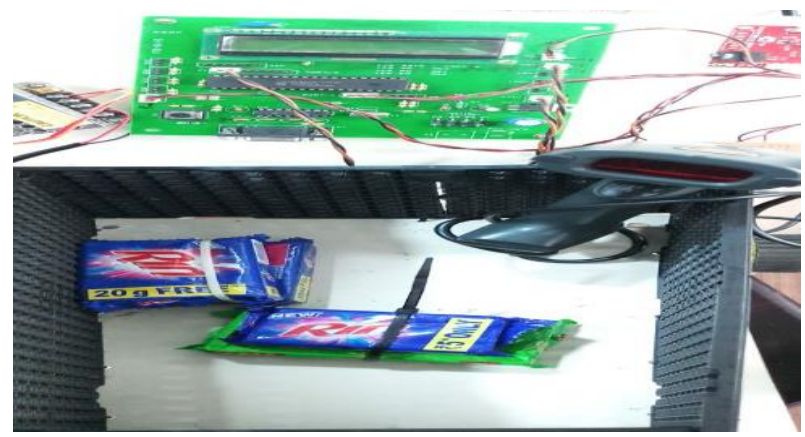

Fig. 7: One of the Product Added without Being Scanned.

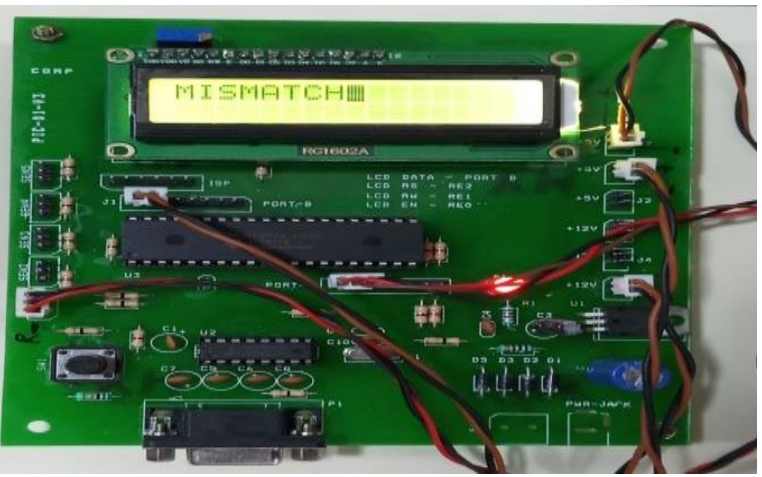

Fig. 8: Mismatch Will Be Displayed As One of Product Added without Being Scanned.

\section{Future scope}

In future, an automatic human and line following shopping trolley with a smart shopping system can be developed. A line following portable robot is installed under the trolley to lead the users to the items' location that they plan to purchase in the supermarket. This involves the hardware and software design of the portable robot. The graphical user interface of Android application during the shopping trolley in operation is expanded. 


\section{Conclusion}

In the modern world, it is necessary to avoid long queues in supermarket shopping which is the biggest issue that people hate in supermarket shopping. To save the time of the customers in supermarket we have introduced SMART TROLLEY to overcome the delay which usually takes place in supermarket billing system especially in special occasions or in festival seasons.

\section{References}

[1] Chih-ChiangWei, National Taiwan University Yi-Ling Chen, National Taiwan University. "Using Mobile Phones to Monitor Shopping Time at Physical Stores" Published in: IEEE Pervasive Computing (Volume: 10, Issue: two, April - June 2011).

[2] W.U.L.J.R. Perera, M. S. Karunarathne "Enhancing And Speeding Up Real-Time-Shopping Using an Indoor Map, Intelligent Suggestions and Calculations, Built Upon a Smart Phone Application" Published in Industrial and Information Systems (ICIIS), 17-20 Dec. 2013.

[3] Yunhao Liu, Yiyang Zhao, Lei Chen "Mining Frequent Trajectory Patterns for activity Monitoring Using Radio Frequency Tag Arrays" Published in: IEEE Transactions on Parallel and Distributed Systems (Volume: 23, Issue: 11, Nov. 2012) Date of Publication: 20 December 2011.

[4] Yu GU, Fuji Ren, Jie Li "PAWS: Passive Human Activity Recognition Based on Wi-Fi Ambient Signals" Published in: IEEE Internet of Things Journal (Volume: three, Issue: five, Oct. 2016).

[5] L. Shang guan et al., "Shop miner: Mining customer Shopping behaviour in physical clothing stores with cots rf Id devices," in Proc. ACM SenSys, Nov. 2015, pp. 113-125.

[6] M. Popa et al., "Analysis of shopping behavior based on Surveillance System," in Proc. SMC, Oct. 2010, pp. 2512-2519.

[7] T. Staake, F. Thiesse, and E. Fleisch, "Extending the EPC Network: The potential of RFID in anti-counterfeiting," in Proc. ACM SAC, Mar. 2005, pp. 1607- 1612 .

[8] J. Han et al., "Cbid: A customer behavior identification System using passive tags," IEEE/ACM Trans. Netw. vol.24, no. 5, pp. 2885-2898, Oct. 2016. 